\title{
New development in near-wall PIV measurements
}

\author{
Hui $\mathrm{Hu}^{1}$, and BoHua Sun ${ }^{2 *}$ \\ ${ }^{1}$ Department of Aerospace Engineering, Iowa State University, Ames 50011, USA; \\ ${ }^{2}$ Department of Mechanical Engineering, Cape Peninsula University of Technology, Cape Town 8000, South Africa
}

Received April 16, 2018; accepted May 15, 2018; published online July 10, 2018

Citation: H. Hu, and B. H. Sun, New development in near-wall PIV measurements, Sci. China-Phys. Mech. Astron. 61, 094731 (2018), https://doi.org/10.1007/s11433-018-9248-x

Very recently, obvious advances have been made in fluid mechanics theoretically [1-6]. The progress of experiment and related method is also worthy of attention. The detailed physics of a near-wall flow is essential for understanding the flow behavior. Very near-wall measurement is widely considered as one of the major technical challenges for fluid mechanics studies, which have a great significance for practical engineering designs and fundamental scientific researches. At present, accurate particle image velocimetry (PIV) measurements very near the wall are still a great challenge. For the measurements near fluid/solid interfaces, Prof. CB Lee and his research group at PKU developed a novel image parity exchange (IPX) technique by adding optimal synthetic particles (OSP) on the solid side in order to get better flow velocity measurements in the near wall region [7]. The improved image-preprocessing method expands the traditional window deformation iterative multigrid scheme in PIV image processing with very large displacement commonly encountered in the measurements of hypersonic boundary layers. Before performing interrogation operation, stationary artificial particles of uniform size are added in the near wall region on the solid side. The initial estimation near the interface is then smoothed by the measurement data from both sides of the interface in order to reduce the large random uncertainties. Interrogations in the following iterative steps converge to the correct results to provide more accurate estimations for particle tracking velocimetries. The algorithm was successfully applied to extract the fine flow structures

*Corresponding author (email: sunb@cput.ac.za) of second-mode waves in a hypersonic boundary layer [8,9] from PIV images with low signal-noise-ratios when traditional PIV methods were found to have technical difficulties, as shown in Figure 1.

The OSP method has also been successfully applied in the experimental investigations of the initial growth of flow asymmetries over a slender body at high angles of attack [10]. Initially different amplitudes of unsteady disturbances near the tip of the slender body are established owing to the tip
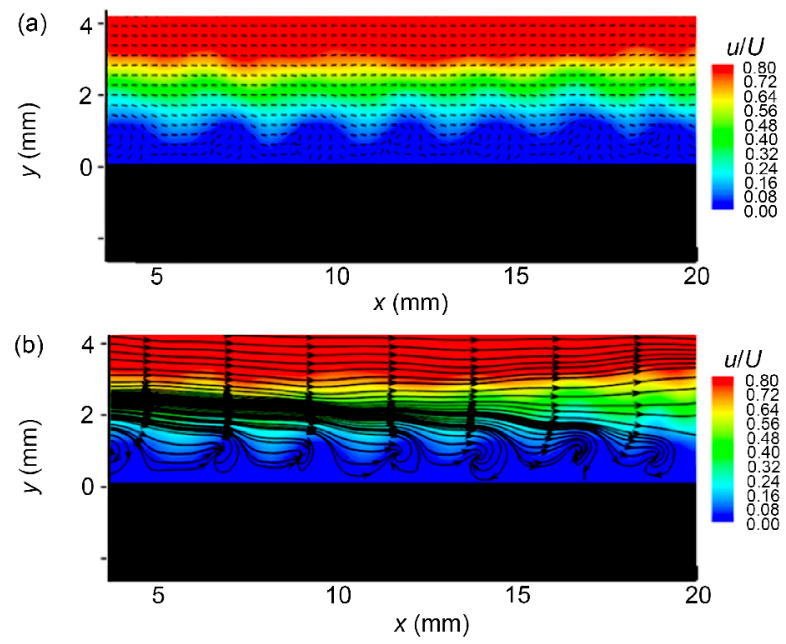

Figure 1 (Color online) Instantaneous PIV results to reveal dynamic flow transition in a hypersonic boundary layer flow with Mach number 6 and free stream velocity $850 \mathrm{~m} / \mathrm{s}$. Second-mode instability waves with typical wavelength twice the boundary layer thickness can be seen. (a) OSP velocity field; (b) OSP streamlines. 
imperfections. The unsteady disturbances experience a super-exponential growth near the tip initially, and then continue to grow exponentially due to linear instabilities. The amplified disturbances lead to vortex of different strengths in the separated shear layers, which feed up continuously the two primary concentrated vortices. The experimental results demonstrate clearly that the initial flow asymmetry arises from an asymmetric development of the boundary layer instability.

Another successful application of the OSP method to characterize the development of second-mode instability in a Mach 6 boundary layer flow over a flat plate when a twodimensional roughness was applied [11]. To the best of the authors's knowledge, it is the first time one observes the evolution of the flow structures of the second-mode instability waves passing through the rough wall experimentally. The experimental results showed clearly that the amplitude of the second-mode instability is first greatly increased upstream of the roughness, then damps and recovers quickly in the vicinity of the roughness, and further downstream acts if as noroughness exists, which confirms Fong's numerical results. The study also indicated that the strength of the amplification and damping is dependent of the roughness height.

The near-wall measurements are even more difficult when the solid wall is moving with curvature, which is typical for turbomachinery flows, especially in the air. The PKU group further proposed a new adaptive stretching of the image strips (AMSI) method [12], which made it possible in measuring the near-wall flows in turbomachines. This is the first successful effort to achieve near-wall measurements over moving interfaces in the air. Figure 2 shows a typical PIV measurement result from the study of the wake's negative jet effect and of wake induced high disturbances near a rotating blade [13]. The obvious changes of the boundary layer development along the suction surface and the existence of flow separation region near the trailing edge of the rotating blade are identified clearly (Figure 2).

With the quantitative information about instantaneous flow fields derived from the PIV measurements, some key advances in wall flow study may be expected. For instance, crucial physical processes related to velocity's first-order derivatives can be accurately analyzed, from which wall friction and dissipation function can be obtained. Note that the hypersonic aerodynamic heating is directly affected by the flow's viscous dissipation function. The recent discovery is reviewed of new principles in aerodynamic heating physics is an encouraging example $[9,14]$, which is crucial to optimal design and safety of hypersonic vehicles $[15,16]$. Finally, we

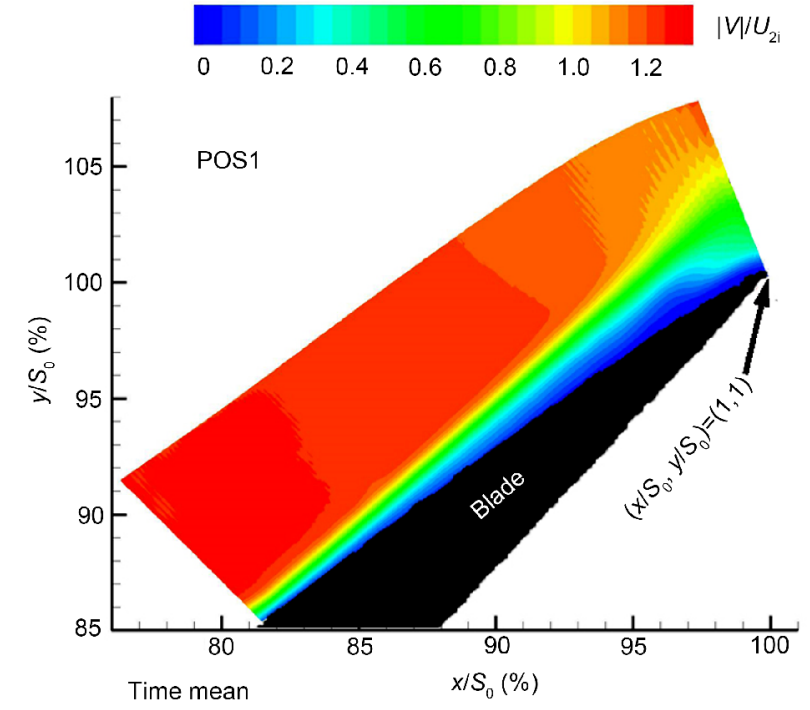

Figure 2 (Color online) The boundary layer over the suction surface of an rotating blade, with its separation depressed by the periodical sweeping of the upstream stator's wake.

hope that in the near future, this new experimental technique will be put in practice, to create high-quality data base for a variety of technologically important wall flows, for testing new concept and theory of wall turbulence $[17,18]$.

1 B. Sun, Appl. Math. Mech.-Engl. Ed. 38, 765 (2017).

2 B. H. Sun, Sci. China-Phys. Mech. Astron. 60, 104711 (2017).

3 Y. C. Hu, W. T. Bi, S. Y. Li, and Z. S. She, Sci. China-Phys. Mech. Astron. 60, 124711 (2017).

4 X. Chen, and Z. S. She, Sci. China-Phys. Mech. Astron. 59, 114711 (2016), arXiv: 1604.08257.

5 J. Z. Wu, Sci. China-Phys. Mech. Astron. 61, 024731 (2018).

6 B. H. Sun, Sci. China-Phys. Mech. Astron. 61, 024721 (2018).

7 Y. Zhu, H. Yuan, C. Zhang, and C. Lee, Meas. Sci. Technol. 24, 125302 (2013).

8 C. Zhang, Y. Zhu, X. Chen, H. Yuan, J. Wu, S. Chen, C. Lee, and M. Gad-el-Hak, AIP Adv. 5, 107137 (2015).

9 Y. Zhu, X. Chen, J. Wu, S. Chen, C. Lee, and M. Gad-el-Hak, Phys. Fluids 30, 011701 (2018).

10 Y. Zhu, H. Yuan, and C. Lee, Phys. Fluids 27, 084103 (2015).

11 Q. Tang, Y. Zhu, X. Chen, and C. Lee, Phys. Fluids 27, 064105 (2015).

12 L. C. Jia, Y. D. Zhu, Y. X. Jia, H. J. Yuan, and C. B. Lee, Meas. Sci. Technol. 28, 035201 (2017).

13 L. Jia, T. Zou, Y. Zhu, and C. Lee, Phys. Fluids 30, 040911 (2018).

14 Y. Zhu, C. Zhang, X. Chen, H. Yuan, J. Wu, S. Chen, C. Lee, and M. Gad-el-Hak, AIAA J. 54, 3039 (2016), arXiv: 1607.00195.

15 B. Sun, and E. S. Oran, Natl. Sci. Rev. 30, (2018).

16 M. Wolverton, Scilight 2018, 010004 (2018).

17 Z. S. She, X. Chen, and F. Hussain, J. Fluid Mech. 827, 322 (2017).

18 C. Lee, and S. Chen, Natl. Sci. Rev. (2018). 